\title{
Grey Relational Grade Based Quantitative Analysis of the Factors Influencing the Load Characteristics of A Power Grid
}

\author{
Wei Pan, Caijia Lei, Wei Jia, Hui Gao, Binghua Fang \\ Guangzhou Power Supply Bureau Co., Ltd., Guangzhou 550002, Guangdong, China
}

\begin{abstract}
Regarding analysis of load characteristics of a power grid, there are multiple factors that influence the variation of load characteristics. Among these factors, the influence of different ones on the change of load characteristic is somewhat different, thus the degree of influence of various factors needs to be quantified to distinguish the main and minor factors of load characteristics. Based on this, the grey relational analysis in the grey system theory is employed as the basis of mathematical model in this paper. Firstly, the main factors affecting the load characteristics of a power grid are analysed. Then, the principle of quantitative analysis of the influencing factors by using grey relational grade is introduced. Lastly, the load of Guangzhou power grid is selected as the research object, thereby the main factor of temperature affecting the load characteristics is quantitatively analysed, such that the correlation between temperature and load is established. In this paper, by investigating the influencing factors and the degree of influence of load characteristics, the law of load characteristics changes can be effectively revealed, which is of great significance for power system planning and dispatching operation.
\end{abstract}

\section{Introduction}

With the rapid development of smart grid technology and smart energy management, especially after the concept of energy Internet was put forward, more and more scholars are focusing on intelligent energy dispatching, management and optimization [1-2], in particular, the dispatching, optimization, and management on load side in the power systems. In the analysis of the load characteristics of power systems, there are many factors that affect the changes in load characteristics, such as [3-4]: economic development level and economic structure, residents' income and living consumption levels, electricity price and temperature climate change, and demand side management measures.

Obviously, a variety of influencing factors work together to determine the trend of power grid load characteristics [5]. Among the many influencing factors, some factors have a great influence on the change of load characteristics, while others have a relatively small impact. Therefore, we need a quantitative analysis of the degree of influence of various factors to distinguish the main and minor factors of load characteristics.

However, in the actual situations, the characteristics and laws of the various types of loads in the power system are not the same, and the causes of their changes are also different and very complex. Therefore, it will be very difficult to mathematically model each influencing factor. . For this reason, in the past, the analysis of the factors that influenced the load characteristics mostly stayed in the qualitative analysis stage and lacked quantitative analysis methods. This made the guidance function of load characteristics analysis not fully utilized.

At present, the correlation analysis method based on mathematical statistics is a relatively common correlation analysis method in relation to the influence factors of load characteristics. Among them, the literatures [6-7] use the correlation method to analyse the relationship between the load and temperature and other factors, which lays a foundation for improving the prediction accuracy of power load. However, these investigations have the following issues to be solved: First, the correlation method used in these literatures requires a large amount of data, which makes it difficult to find real statistical rules from a small amount of data. Second, the method requires the influencing factors and system characteristics. There is a linear relationship between the data.

In recent years, the grey relational analysis has been introduced as a statistical analysis technique by scholars into the research of power system-related problems [8-9]. For instance, grey relational analysis has extensive applications in power system load forecasting and impact load correlation analysis [10-13]. Grey relational grade analysis is an effective method to analyse the correlation degree of various factors in the system. It can analyse the correlation degree and model construction of various influencing factors of the system when the system is unclear and the data is incomplete. Taking the load forecasting as an example, the application of grey

\footnotetext{
*Corresponding author: Wei Pan, kddk_scut@sina.com
} 
correlation approaches to power system load forecasting helps to improve the accuracy of load forecasting. It can also be used to quantitatively analyse the factors affecting the load characteristic index.

Based on this, the grey correlation analysis in grey system theory is used as the mathematics foundation of modelling, and the load of Guangzhou Power Grid is selected as the research object. The main influencing factors of load characteristics are qualitatively analysed and calculated to obtain the law of change of load characteristics. In addition, this paper establishes a comprehensive quantitative analysis model of the factors affecting the change of load characteristics. This paper makes a comprehensive analysis and verification of the factors affecting the load of Guangzhou Power Grid based on the grey relational degree theory. By studying the influencing factors and the degree of influence of load characteristics, the law of load characteristics changes can be effectively revealed, which is of great significance for power system planning and dispatching operations.

\section{Main factors influencing the load characteristics of power grid}

Through investigation and literature analysis, we found that the main influencing factors affecting the load characteristics include the following aspects:

- The level of economic development and adjustment of industrial structure;

- Improvement of income level and living standard, and changes in consumer attitudes;

- Electricity price, such as time-of-use price;

- Major holidays;

- Power supply side;

- Demand side management measures;

- Weather conditions.

The impact of the above factors on the load characteristics is briefly analysed as follows.

\subsection{Influencing of economic development level and industrial structure adjustment}

Regional economic development not only determines the growth rate of electricity load, but also has a greater impact on the characteristics of the electricity load. This effect is mainly reflected in changes in the load characteristics caused by changes in the proportion of electricity used in various industries.

In recent years, the trend of load changes in various industries has the following performance: In recent years due to the influence of climate, the fluctuations in electricity consumption in the primary industry have been large. However, in general, the primary industry's electricity consumption shows a trend of low growth. Due to the impact of the economic climate, the growth rate of the overall electricity load of the secondary industry was slow, far below the average growth level of the whole society. The level of electricity used by the tertiary industry and residents has increased rapidly, and the proportion of electricity consumption in the entire society has gradually increased.

In general, industrial users have high levels of load, and the first and third industries and households have lower levels of electricity load. Therefore, the greater the proportion of industry in the industrial structure, the higher the load rate level will be. The larger the proportion of electricity consumed by the primary and tertiary industries and residents, the more they will be able to reduce the load rate.

\subsection{Influencing of income level and living standard improvement, and consumer attitude change}

The increase in income levels and living standards is closely related to the level of economic development. With the rapid development of the economy, the society continues to improve, and the people's income level and material and cultural living standards are also continuously improving. The household ownership rate of households, especially urban residents, has grown rapidly. The impact of household electricity load on load characteristics is mainly reflected in the increase in seasonal load components of the grid due to the increase in household appliances such as air-conditioners, directly affecting the size of the system peak load and the time of occurrence.

With the increasing of residents' income, the popularity of household appliances, especially airconditioning appliances, has increased seasonal load components. In addition, because the tertiary industry is mainly a service unit such as finance, restaurants, shopping malls, and hotels, its load is, to a large extent, seasonal electrical equipment such as air conditioners and fans that are closely related to the season. Therefore, further development of the tertiary industry will also cause an increase in the seasonal load component of the total load.

In addition, because the air-conditioning and other electrical equipment are basically used in the same time, which makes the rapid increase in peak load of the power grid, peak and valley difference is getting larger and larger.

\subsection{Influencing of electricity price}

As the link between the power supply and the power demand, electricity prices obviously have a certain impact on electricity demand. With the gradual increase in the degree of marketization, the impact of electricity prices on electricity demand has become increasingly evident. The main performance is as follows:

First, the level of electricity consumption affecting business and industry, when the electricity price is higher than the enterprise's capacity, the electricity consumption will be reduced;

Second, the distribution of high energy-consuming industries affects the development of the region, high energy-consuming companies to consider the cost factor, from the regions with high electricity prices have moved 
to areas with low electricity prices, affecting the pattern of growth in electricity demand.

In addition, the implementation of the peak-to-valley electricity price can, on the one hand, suppress the growth of peak load, and on the other hand also play a significant role in increasing the load rate, which is an effective measure to improve the load characteristics of the power grid.

\subsection{Influencing of major holidays}

With the implementation of the long vacation system, the Spring Festival, May Day and National Day each year have a significant impact on the power load. Especially after implementing the new holiday in 2008, the impact on the load was even more pronounced. During long holidays, many industrial loads are stopped or reduced, and the shape of their load curves is quite different from that of ordinary ports.

Generally speaking, the average load rate and the average valley and valley difference of the Spring Festival of each year are lower than those of ordinary working hours. This is because, during the Spring Festival period, the electricity consumption is large, and the late peak load is large. The low valley is not obvious. The flat section load is not much higher than the valley load, which leads to a lower average load, and reduces the period of the Spring Festival, and the average load rate and the average peak and valley difference.

The average load rate of Ching Ming Festival, Dragon Boat Festival, May Day, National Day and MidAutumn Festival is not much different from the average load rate of the month in which these festivals and holidays were held. The average valley and valley difference is lower than these holidays. The difference between the peaks and valleys in the month is small. This is because people's resorts are not the same, electricity is relatively dispersed, and the electricity load is more balanced than usual. The peak load is not much higher than the flat load.

\subsection{Influencing of power supply side}

When electricity is in short supply, the impact of power cuts on load characteristics is mainly reflected in:

- Changing the shape of the daily load curve during the peak load period can generally reduce the daily peak load and increase the daily load rate.

- If power cuts occur on the maximum load day of the month, the shape of the annual load curve will be affected.

\subsection{Influencing of demand side management measures}

Strengthening the power demand side management through the implementation of peak-valley electricity prices, signing with the user to transfer the peak load agreement, promotion of energy storage equipment and other measures, can effectively avoid peak power and ease grid pressure. The implementation of peak-to-valley tariffs can encourage end-users who can avoid peak hours to use electricity to reduce electricity bills and try to use electricity during low times to achieve the purpose of power load peaking and valley filling. Through peak load shifting, electricity consumption is limited at some time in different industries in order to avoid overconcentration of electricity demand over a certain period of time, resulting in a serious shortage of electricity supply.

Therefore, demand-side management can play a positive role in improving the load characteristics. Through peak load shifting, the maximum load of the grid can be reduced, the load rate of the grid can be increased, and the peak-to-valley ratio of the grid can be reduced. The influence of the peak shift on the load characteristics is also reflected in the change in the shape of the load curve, which can reduce the peak load.

\subsection{Influencing of weather conditions}

Many studies have shown that load is closely related to meteorological conditions, in addition to seasonality, time, and various user activities. In summer, when temperatures are high, people have to cool down by turning on air conditioners, fans, etc.; in winter, the temperature is low, people use various means to heat the weather; when the weather is dark, people use the lighting equipment to illuminate; when the humidity is high, the humidity is relatively high. Departments with higher requirements must change the local humidity conditions through dehumidification equipment. These measures will inevitably increase the power load.

The meteorological factors affecting the load change include temperature, humidity, wind speed, atmospheric pressure, precipitation, and cloudiness, among which the air temperature has the greatest impact on the load. In practical research, generally consider the comprehensive effects of meteorological factors on the load, such as the wet temperature index, human comfort index, etc.

\section{Quantitative analysis method for influencing factors}

\subsection{Grey relational grade analysis}

Grey relational grade analysis is based on the degree of similarity of the geometric shape of the behavioral sequence curve to determine whether the contact is tight. The closer the curve is, the greater the correlation between the corresponding sequences. It is an analysis method to determine the extent of influence between factors or the contribution of factors to the main behavior. The purpose of the grey relational grade analysis is to find the main relationship between various factors, so as to determine which is the relevant behavioral factor that cannot be ignored, and to find out the degree of correlation of each relevant influencing factor to the main behavior in order to grasp the main contradiction in the development of the thing. Therefore, it is particularly suitable for the analysis and evaluation 
of this data-limited, unprototypical, complex and uncertain problem.

As a statistical analysis technology, the grey relational grade analysis is an effective method to analyze the degree of association of various factors in the system. At present, in the correlation analysis, regression analysis, variance analysis, principal component analysis, and data envelopment analysis (DEA) in mathematical statistics are methods used to perform system correlation analysis. However, these analysis methods have the following deficiencies:

- It requires a large amount of data and a small amount of data to find statistical rules.

- The sample is required to obey a typical probability distribution. It requires a linear relationship between each factor data and the system characteristic data, and each factor has nothing to do with each other. This requirement is often difficult to meet.

- The amount of calculation is large and generally depends on the help of a computer.

- Sometimes the quantification result may not be consistent with the qualitative analysis result, resulting in distortion and reversal of the system's relations and laws.

The grey relational grade analysis makes up for the defects caused by the use of mathematical statistics methods for system analysis. It is equally applicable to the number of sample sizes and the presence or absence of samples in the system, and the calculation is small and convenient, and there is no case where the quantitative results are inconsistent with the qualitative analysis. For this reason, this paper introduces the theory of grey relational grade analysis in the influence factors of load characteristics to carry out comprehensive quantitative analysis.

\subsection{Specific implementation procedures}

The specific process of this grey relational grade analysis method is shown in the Figure 1 below.

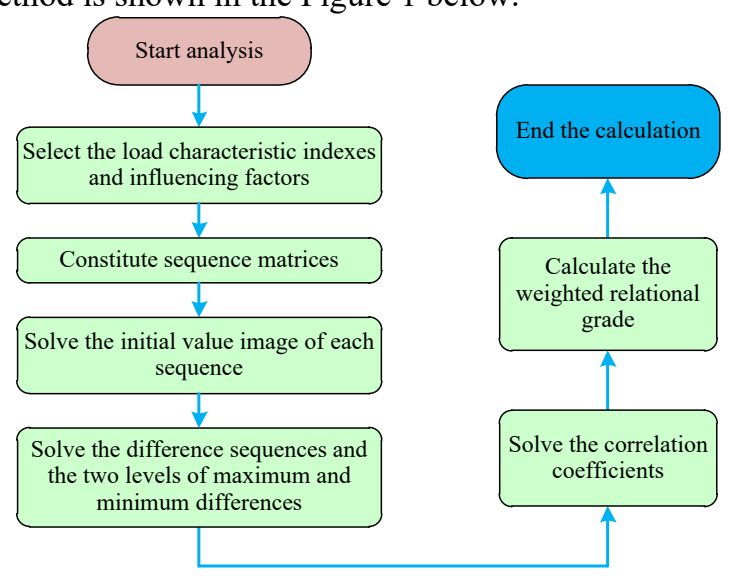

Fig. 1. The flow chart of grey relational grade analysis.

Based on Figure 1, the main procedures involved in the grey relational grade analysis are introduced as follows.

(1) Selecting of main load characteristic indexes

Among the factors that affect the load characteristics change, the selection of the main load characteristics is based on the in-depth analysis of the load characteristics of the power system, based on its role in the power system scheduling operation, planning, and marketing.

a) Annual maximum load

This indicator is important for grid planning. From the perspective of planning, the annual maximum load is an extremely important parameter. The annual maximum load in the planning period is an important basis for the installed capacity, power supply structure and investment scale of the decision system.

b) Annual maximum load utilization hours

The maximum load utilization hours of the year is mainly reflected in the time utilization efficiency of measuring load and the increase or decrease in the annual maximum load utilization hours caused by different proportions of electricity consumption in various industries. For example, when the proportion of the secondary industry is large, the annual maximum load utilization hour is relatively high. When the proportion of the tertiary industry and the household electricity consumption is relatively large, the annual maximum load utilization hours will decrease.

c) The maximum peak-to-valley difference

The year's maximum peak-to-valley difference can help dispatchers rationally arrange peak load standby capacity and improve the economic efficiency of the grid operation.

d) Annual load rate

The annual load rate reflects the effect of various types of power structure changes on the shape of the load curve. The load rate will increase with the increase in the proportion of electricity used by the secondary industry, and will decline as the proportion of electricity consumed by the tertiary industry and residents increases.

Therefore, the annual maximum load, annual maximum load utilization hours, annual maximum peakto-valley difference, and annual load rate can be selected to compose the main feature sequence.

(2) Selecting of major influencing factors

In the analysis of the factors affecting load characteristics, the main purpose of the grey correlation analysis of the factors that affect the characteristics of the load is to have a comprehensive quantitative assessment of the impact of these factors on the change of load characteristics. The impact of economic development on changes in load characteristics is mainly reflected in the total national economy and various industrial structures, and the industrial structure also influences changes in load characteristics through its electricity consumption structure; social development is mainly reflected in the urbanization rate and per capita disposable income, and so on. Therefore, the economic structure, electricity structure, electricity price, air temperature, and social factors can be selected to constitute the sub-sequences that affect the load characteristics change.

(3) Constitute the sequence matrices

When the quantitative analysis of the object sequence (system feature sequence) and influencing factor sequence (sub-sequence) is more than one, the system feature sequence can be represented by $Y_{j}$, and the 
related factor subsequence is represented by $X_{0}, X_{1} \ldots, X_{i}$. The sequence matrix formed is:

$$
\left(Y_{j}, X_{0}, X_{1} \ldots, X_{i}\right)=\left[\begin{array}{cccc}
y_{j}(0) & x_{0}(0) & \cdots & x_{i}(0) \\
y_{j}(1) & x_{0}(1) & \cdots & x_{i}(1) \\
\vdots & \vdots & \ddots & \vdots \\
y_{j}(t) & x_{0}(t) & \cdots & x_{i}(t)
\end{array}\right]
$$

where $t=0,1, \cdots 5 ; Y_{j}$ refers to the value of annual characteristic sequence; $X_{i}$ represents the value of annual influencing factor sequence.

(4) Solve the initial value image of each sequence

Before analyzing an abstract system or phenomenon, we must first select the data sequence that reflects the characteristics of the system behavior, which is called the mapping amount of system behavior. The mapping amount is used to indirectly characterize the system behavior. After selecting the mapping quantity of system behavior characteristics, it is necessary to further clarify the effective factors that affect system behavior. Different sequences may have very different values. Even the dimensions are not comparable. Therefore, they must first be standardized. This paper uses the first normalization process to facilitate comparison. The formula is as follows:

$$
\left\{\begin{array}{l}
x_{i}^{\prime}(t)=\frac{x_{i}(t)}{x_{i}(0)} \\
y_{j}^{\prime}(t)=\frac{y_{j}(t)}{y_{j}(0)}
\end{array}\right.
$$

where $i, j=0,1, \cdots, m$.

(5) Solve the difference sequence

If all the factors in the system factor set are considered as spatial points, and the observation data of each factor for different moments, different indexes, and different objects are regarded as the coordinates of the points, the factors can be studied in a specific twodimensional space. The relationship between the inter-or factor and system characteristics, the formula for calculating the difference sequence is as follows:

$$
\Delta_{j i}(t)=\left|y_{j}(t)-x_{i}(t)\right|
$$

where $t=0,1, \cdots 5$.

Then, we solve the two-levels of maximum and minimum differences as

$$
\left\{\begin{array}{l}
M=\max _{i} \max _{t} \Delta_{j i}(t), \text { maximum difference } \\
m=\min _{i} \min _{t} \Delta_{j i}(t), \text { minimum difference }
\end{array}\right.
$$

(6) Solve the corresponding coefficient

The correlation coefficient is mainly used to calculate the correlation between the main feature sequence and the influencing factor subsequence at different time periods. The calculation formula is as follows:

$$
\lambda_{j i}(t)=\frac{m+\rho \cdot M}{\Delta_{j i}(t)+\rho \cdot M}
$$

where $t=0,1, \cdots 5 ; \rho \in(0,1)$ is the discrimination coefficient, which is generally taken 0.5 .

(7) Calculate the grey relational grade

In order to calculate the degree of correlation between the main feature sequence and the influencing factor sub-sequence, the correlation coefficient of each time period must be weighted and summed to obtain the degree of correlation between them. The formula is as follows:

$$
r_{j i}=\sum_{t=0}^{5} \omega(t) \lambda_{j i}(t)
$$

where $t=0,1, \cdots 5 ; \omega(t)$ is the weight of each year.

\section{Grey relational grade based analysis for the temperature and load in the Guangzhou Power Grid}

In recent years, as the country's modernization progresses with each passing day, people's living standards have increased and the country's economy (especially the tertiary industry) has grown rapidly. The ownership and use of cooling equipment and heating equipment have gradually increased, driving meteorologically sensitive loads ( Such as cooling load and heating load, which is very sensitive to climate change load, the proportion continues to rise. Therefore, it is necessary to study the correlation between air temperature and load, and accurately grasp the influence of temperature on load characteristics.

Taking the daily maximum load and daily maximum temperature data for July and August (summer) in a certain area as an example, the correlation between them is analyzed. According to the collected data, draw a scatter plot and fit it to get the fitting result, as shown in Figure 2.

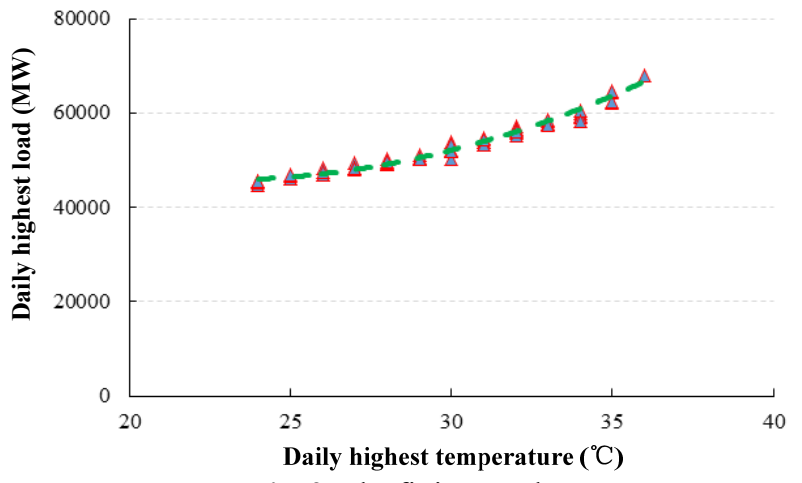

Fig. 2. The fitting result.

As can be seen from the figure, the maximum daily load and daily maximum temperature in the region show a strong correlation. When the temperature is around 25 degrees Celsius, the increase in the daily maximum temperature will cause the daily maximum load to increase slightly. When the temperature exceeds 30 degrees, the maximum daily load will increase significantly with the increase of the daily maximum temperature, indicating that the area contains more cooling load during the summer, when the temperature rises to a certain extent, the cooling load increases significantly, leading to the The maximum load increases.

According to the introduction of the correlation degree analysis method above, the correlation degree between the daily maximum load and the daily maximum temperature is calculated, and the correlation degree is found to be 0.8827 , indicating that there is a 
strong positive correlation between daily maximum load and daily maximum temperature in the area. In order to better describe the law of daily maximum temperature change with the maximum daily load, all load values corresponding to the same temperature are averaged to form a sequence of one-to-one correspondence between temperature and daily maximum load, and a piecewise linear fitting method is used. A one-dimensional linear function of the maximum daily load change with temperature in the region during the summer was fitted, and 30 degrees Celsius were used as segment points to fit into two straight lines. The fitting results are presented as $y=1116.5 x+44112$ when $x \in[24,30]$, and $y=2471.4 x+48864$ when $x \in[30,36]$.

Based on the fitting results, it can be seen that when the temperature is between 24 and 30 degrees, the daily maximum load is increased by $1116.5 \mathrm{MW}$ for each degree of increase. When the temperature is between 30 and 36 degrees, the maximum daily load is approximately increased by $2471.4 \mathrm{MW}$.

\section{Conclusion}

This paper conducts the follow investigations: first, we conduct a comprehensive analysis of factors affecting load characteristics, and the specific influencing factors include economic development levels, residents' income levels, electricity prices, major holidays, electricity supply testing, and demand-side management measures; second, based on the mathematical theory of grey system, we establish a quantitative analysis model of the influencing factors of load characteristics.

By studying the influencing factors and the degree of influence of load characteristics, the law of load characteristics changes can be effectively revealed, which is of great significance for power system planning and dispatching operations.

\section{Acknowledgement}

The work is partially supported by the Science and Technology Projects of China Southern Power Grid.

\section{References}

1. L.F. Cheng, T. Yu, X.S. Zhang, et al. Cyberphysical-social systems based smart energy robotic dispatcher and its knowledge automation: framework, techniques and challenges. Proceedings of the CSEE, 2018, 38(1): 25-40.

2. L.F. Cheng, Z.Y. Zhang, H.R. Jiang, et al. Local energy management and optimization: A novel energy universal service bus system based on energy Internet technologies. Energies, 2018, 11(5), 1160.

3. Z. Wang, S. Bian, Y. Liu, et al. The load characteristics classification and synthesis of substations in large area power grid. International Journal of Electrical Power \& Energy Systems, 2013, 48(1):71-82.
4. X. Xu, J. Xi, Y. Li, et al. Research on the dynamic frequency characteristic of large-scale power grid considering the action of power system splitting and load shedding. In proceedings of the International Conference on Power System Technology, 2014, 103-108.

5. L.I. Hu, Q. Zhou, J. Shi, et al. Impact of large-scale electric vehicles connected to power grid on the load characteristics in nanjing city and the corresponding countermeasures. Proceedings of the CSU-EPSA, 2016.

6. Y.G. Huang, Y. Lu. Characteristic analysis and forecasting of summer daily peak load. Power DSM, 2009, 11(4): 35-37.

7. Y.F. Yin. Analysis on impact of air temperature on local load characteristics. Guangdong Electric Power, 2011, 24(10): 79-83.

8. L.F. Cheng, T. Yu, G.P. Wang, et al. Hot spot temperature and grey target theory-based dynamic modelling for reliability assessment of transformer oil-paper insulation systems: A practical case study. Energies, 2018, 11(1), 249. DOI:10.3390/ en1 1010249

9. L.F. Cheng, T. Yu. Dissolved gas analysis principlebased intelligent approaches to fault diagnosis and decision making for large oil-immersed power transformers: A survey. Energies, 2018, 11(4), 913. DOI:10.3390/en11040913

10. W.Y. Liu, D.Y. Men, J.F. Liang, et al. Monthly load forecasting based on grey relational degree and least squares support vector machine. Power System Technology, 2012, 36(8): 228-231.

11. L.F. Cheng, B. Zhou, D.H. Cai, et al. Lifetime assessment and optimized maintenance system of transformers based on the HST model. Lecture Notes in Electrical Engineering, v 334, p 417-430, 2015. DOI: 10.1007/978-3-319-13707-0_46

12. Y.J. Zhang, H. Shi. Distribution network energysaving investment compact planning based on grey connectedness weighting. Automation of Electric Power Systems, 2010, 34(22): 46-50.

13. B. Song, Y. Ping, Y. Luo, et al. Study on the fault diagnosis of transformer based on the grey relational analysis. In Proceedings of the International Conference on Power System Technology, 2002. Proceedings. Powercon. IEEE, 2002, 4: 2231-2234. 